\title{
Detecting Microbially Induced Calcite Precipitation in a Model Well-Bore Using Downhole Low-Field NMR
}

\author{
Authors: Catherine M. Kirkland, Sam Zanetti, Elliot \\ Grunewald, David O. Walsh, Sarah L. Codd, \& \\ Adrienne J. Phillips
}

This document is the unedited author's version of a Submitted Work that was subsequently accepted for publication in Environmental Science \& Technology, copyright ( A American Chemical Society after peer review. To access the final edited and published work, see 10.1021/ acs.est.6b04833.

Kirkland CM, Zanetti S, Grunewald E, Walsh DO, Codd SL, Phillips AJ, "Detecting Microbially Induced Calcite Precipitation in a Model Well-Bore Using Downhole Low-Field NMR," Environmental Science \& Technology, 2017 Feb 7;51(3):1537-1543. doi: 10.1021/ acs.est.6b04833. 


\title{
Detecting Microbially Induced Calcite Precipitation in a Model Well- Bore Using Downhole Low-Field NMR
}

\author{
Catherine M. Kirkland, Sam Zanetti, Elliot Grunewald, David O. Walsh, Sarah L. \\ Codd, and Adrienne J. Phillips
}

\begin{abstract}
Microbially induced calcite precipitation (MICP) has been widely researched recently due to its relevance for subsurface engineering applications including sealing leakage pathways and permeability modifica-tion. These applications of MICP are inherently difficult to monitor nondestructively in time and space. Nuclear magnetic resonance

(NMR) can characterize the pore size distributions, porosity, and permeability of subsurface formations. This investigation used a low-field NMR well-logging probe to monitor MICP in a sand-filled bioreactor, measuring NMR signal amplitude and T2 relaxation over an 8 day experimental period. Following inoculation with the ureolytic bacteria, Sporosarcina pasteurii, and pulsed injections of urea and calcium substrate, the NMR measured water content in the reactor decreased to $76 \%$ of its initial value. T2 relaxation distributions bifurcated from a single mode centered about approximately 650 $\mathrm{ms}$ into a fast decaying population (T2 less than $10 \mathrm{~ms}$ ) and a larger population with $\mathrm{T} 2$

greater than $1000 \mathrm{~ms}$. The combination of changes in pore volume and surface

minerology accounts for the changes in the $\mathbf{T} 2$ distributions. Destructive sampling confirmed final porosity was approximately $88 \%$ of the original value. These results indicate the low-field NMR well-logging probe is sensitive to the physical and chemical changes caused by MICP in a laboratory bioreactor.
\end{abstract}

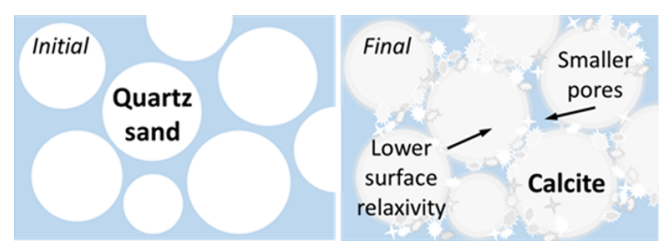

\section{INTRODUCTION}

Biofilms form when bacteria secrete a matrix of extracellular polymeric substance (EPS), attaching themselves to solid surfaces in colonies akin to multicellular organisms and buffering their microscale environment. ${ }^{1}$ Bacterial biofilms are known to induce metal corrosion, ${ }^{2}$ cause persistent infections, ${ }^{3}$ treat wastewater, ${ }^{4}$ or remediate contaminated groundwater. 5 When composed of ureolytic microbes, biofilms can also induce calcite precipitation, ${ }^{6,9}$ a process referred to as biomineralization or microbially induced calcite precipitation (MICP). Many strains of bacteria found naturally in soil and groundwater are ureolytic, meaning they can hydrolyze urea for energy and a source of nitrogen. ${ }^{7}$ Catalyzed by the microbially produced urease enzyme, water cleaves urea to form ammonia and carbon dioxide which, due to a $\mathrm{pH}$ increase, shifts the carbonate equilibrium toward bicarbonate and carbonate. Then, with sufficient calcium and carbonate activity, calcium carbonate ( $\mathrm{CaCO}_{3}$ or calcite) precipitates (eq 1).

$$
\left(\mathrm{NH}_{2}\right)_{2} \mathrm{CO}+2 \mathrm{H}_{2} \mathrm{O}+\mathrm{C}_{\mathfrak{Z}}^{\dagger} \Rightarrow 2 \mathrm{NH}_{4}^{+}+\mathrm{CaCO}_{3}(\mathrm{~s})
$$

Sporosarcina pasteurii, the ureolytic bacteria used in this experiment, forms a thin biofilm in porous media ${ }^{10}$ where the EPS matrix, a 3-dimensional diffusion-limited hydrogel, can either facilitate or inhibit MICP over microscales. The organic molecules comprising the EPS matrix restrict mass transfer, creating localized chemical gradients within the hydrogel structure. ${ }^{6} \mathrm{Ca}^{2+}$ ions are not used in metabolic processes and accumulate near cell surfaces where ureolysis produces an alkaline environment. Thus, the microbial biofilm matrix provides nucleation sites for calcite precipitation. 11 In porous media, the precipitated calcite binds together media grains and fills pore spaces. 12

MICP has engineering applications 8 that include soil stabilization 12,13 and subsurface barriers, ${ }^{14}$ sealing of cap rocks and well-bore regions for carbon dioxide sequestration, 15-17 and limestone and concrete remediation. 11 Many of these beneficial applications of MICP occur in the subsurface, raising the question of how the process can best be monitored spatiotemporally. Nuclear magnetic resonance (NMR) is commonly used nondestructively and noninvasively to characterize the pore size distributions, porosity, and permeability of subsurface geologic formations. 18 These are the same physical properties affected by MICP, indicating that NMR well-logging tools may have potential for monitoring 
subsurface engineering applications of MICP. This study used a low-field NMR well-logging tool designed for subsurface hydrogeologic investigations ${ }^{19}$ to detect changes in NMR signal response indicative of MICP in the pore spaces of sandfilled radial-flow bioreactor.

Background. There are limited examples in the scientific literature where NMR methods have been applied to the study of biomineralization in porous media relevant for engineering applications. ${ }^{10,20}$ These previous studies have used high field strength magnetic resonance imaging (MRI) along with other NMR methods to probe hydrodynamic properties of biomineralization in model porous media systems.

Fridjonsson et al. ${ }^{10}$ used high-field NMR to measure changes in hydrodynamic dispersion resulting from MICP in model porous media to compare flow dynamics between systems influenced by either solid precipitates or a biofilm matrix. The authors used a combination of NMR displacement measurements, relaxation mapping, MRI, and microscopy methods.

Sham et al. ${ }^{20}$ used MRI and NMR flow measurements on both a model bead pack and a Bentheimer sandstone rock core to examine structure and transport properties of each system following MICP. The authors report a reduction of $3.7 \%$ in absolute porosity in the bead pack, which correlated to a $98 \%$ reduction in permeability. In the sandstone, a $7.2 \%$ reduction in absolute porosity yielded a $96.5 \%$ reduction in permeability. In both systems, preferential fouling of the inlet region of the column was observed.

The low-field NMR well-logging tool used in this study (Javelin JP350, Vista Clara, Inc., Mukilteo, WA) is sensitive to biofilm growth in the pore spaces of a sand-filled bioreactor ${ }^{21}$ and in the subsurface soil of an engineered field testing site. ${ }^{22}$ In both of these studies, biofilm growth caused enhanced relaxation with $T_{2}$ relaxation times decreasing by approximately $40-60 \%$.

These previous studies show (1) NMR methods are useful for analyzing changes resulting from MICP in porous media and (2) the well-logging tool is sensitive to small changes over time in the microscale pore environment. To our knowledge, field scale low-field NMR instruments have not been applied to the measurement or monitoring of MICP. In the current study, $\mathrm{CaCO}_{3}$ precipitation was expected to change the NMR signal response by reducing the liquid fraction from which the signal is obtained, causing a decrease in signal amplitude over time as the pores accumulate calcite. MICP will also change the pore sizes and mineral surface of the porous media, thereby influencing the signal relaxation response. A correlation between the signal response and reduction of porosity due to MICP may indicate the use of a NMR well-logging tool as a sensor for biomineralization in field applications where optical or destructive monitoring methods are not possible. This study represents a first step toward that end by demonstrating that a NMR well-logging tool is sensitive to MICP.

NMR Theory. The NMR well-logging tool is sensitive to the hydrogen protons in water, called "spins", such that the behavior of the NMR signal over time is related to the various microscale water environments in the surrounding formation. The tool measures $1.37 \mathrm{~m}$ long and $8.9 \mathrm{~cm}$ in diameter and is designed to be lowered into small-diameter cased or uncased borehole wells (Figure 1). ${ }^{19}$ The dual frequency probe used in this experiment operates at approximately 250 and $300 \mathrm{kHz}$ and is composed of an array of permanent magnets and radio frequency (RF) induction coils. ${ }^{19}$ The permanent magnets establish a static magnetic field, $\mathbf{B}_{0}$, along the direction of the

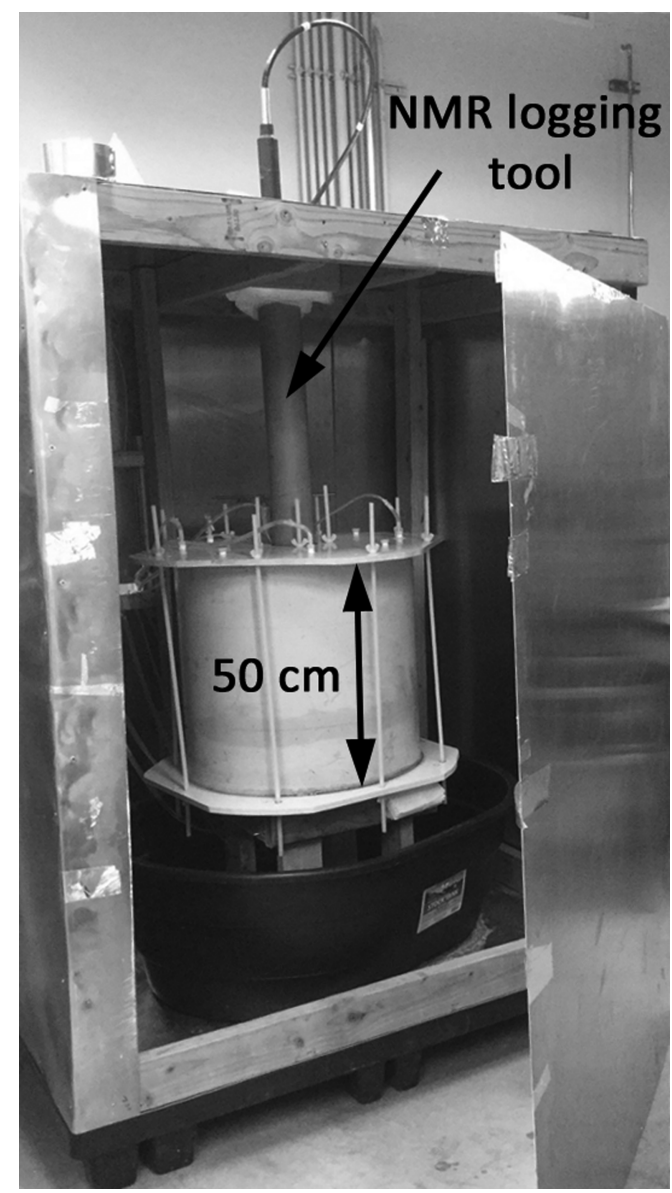

Figure 1. Radial flow bioreactor and NMR logging tool were housed in a Faraday cage to reduce detection of electromagnetic noise from the laboratory.

borehole, where the field strength depends on the radial distance from the tool. The RF pulses produce $2 \mathrm{~mm}$-scale cylindrical excitation shells at radial distances of $17-19 \mathrm{~cm}$ from the probe center and in the middle of the reactor's sand annulus. The excited shells are $50 \mathrm{~cm}$ in height. Only spins in these two excitation shells contribute to the measured NMR signal response, which is averaged over all the spins in each shell.

The initial amplitude of the NMR signal is proportional to the amount of water in the excitation shell and reflects the volumetric water content, or porosity, of the porous media. The NMR signal amplitude decreases when water is displaced by mineral formation in the pores.

The observed decay rate reflects spin-spin, or $T_{2}$, relaxation, which occurs as protons interact with each other in the transverse plane. These interactions cause a dephasing of spin coherence and signal attenuation. In geologic materials, the observed $T_{2}$ relaxation rate comprises the bulk relaxation rate of the pore fluid, $\frac{1}{T_{2 B}}$, the surface relaxation rate, $\frac{1}{T_{2 S}}$, related to interactions between the fluid and the pore walls, and the diffusion relaxation rate, $\frac{1}{T_{2 D}}$, related to diffusion of fluids within pores due to inhomogeneities in the local magnetic field (eq 2)..$^{23,24}$

$$
\frac{1}{T_{2}}=\frac{1}{T_{2 \mathrm{~B}}}+\frac{1}{T_{2 \mathrm{~S}}}+\frac{1}{T_{2 \mathrm{D}}}
$$


At the low magnetic field strength used in this study, the experimental parameter of the echo spacing, $t_{\mathrm{E}}$, can be selected to make the influence of diffusion relaxation, $T_{2 \mathrm{D}}$, sufficiently small to be neglected. ${ }^{21,24}$ For the current study, changes in the fluid properties of the pore liquid, such as viscosity, are not expected be a significant factor in the overall change of the system $T_{2}$ relaxation time. ${ }^{10}$ The influence of changes in $T_{2 \mathrm{~B}}$ can therefore also be neglected. Changes in surface relaxation, $T_{2 S}$, are expected to dominate changes in the observed $T_{2}$ of this experimental system.

The low-field NMR signal response in most saturated natural geologic media is dominated by surface relaxation. ${ }^{23,25}$ Surface relaxation occurs as excited spins approach and interact with the pore walls. Thus, the rate of surface relaxation is most strongly related to pore size and the mineral surface of the solid matrix. Surface relaxation occurs faster in small pores with a high surface-area-to-volume-ratio because the diffusing water molecules are more likely to interact with the grain surface. The surface relaxation rate also depends on the propensity of the surface for inducing relaxation, a characteristic referred to as surface relaxivity, $\rho$. Greater concentrations of paramagnetic ions like $\mathrm{Fe}^{3+}$ and $\mathrm{Mn}^{2+}$ produce higher magnitudes of $\rho$ and faster relaxation rates. ${ }^{26,27}$ In heterogeneous materials with a range of pore sizes or variable $\rho$, there may be a distribution of relaxation rates making up the bulk response. Thus, an Inverse Laplace Transform yields a decay-time distribution that can be interpreted as a distribution of pore environments.

In our experiments, we expect MICP to have several combined influences on the NMR response. First, we expect that growth of calcite within the pore space will reduce the total porosity and water content. We also expect the growth of $\mathrm{CaCO}_{3}$ to influence the observed relaxation rate due to changes in mineralogy and pore size. ${ }^{28-30}$ The quartz sand used in this study is coarse-grained and contains small percentages of paramagnetic species including iron oxide $\left(\mathrm{Fe}_{2} \mathrm{O}_{3}\right)$ at a mean weight percent of 0.04 (2095 Granusil silica sand, Unimin Corp., Ottawa, $\mathrm{MN}$ ). We expect that $\mathrm{CaCO}_{3}$ forming on the quartz grain surfaces will decrease the total macropore dimension which could drive faster relaxation rates. On the other hand, $\mathrm{CaCO}_{3}$ precipitating on the grain surface may shield water from the paramagnetic ions on the sand, thus decreasing the average $\rho$ of the grain surface. A lower average $\rho$ would tend to decrease the surface relaxation rate in the macropores, resulting in longer overall $T_{2}$. Further, the $\mathrm{CaCO}_{3}$ may form microcrystalline structures that incorporate significant microporosity of nanometer scale. We expect water in the very small geometry of these micropores to exhibit very short relaxation times. Thus, we anticipate these changes in the pore structure concurrent with MICP will manifest themselves as multiple changes to the NMR $T_{2}$ relaxation time distribution. These observed changes are expected to indicate which mechanism dominates in the bioreactor where there exists a particular initial pore size distribution and surface minerology.

\section{MATERIALS AND METHODS}

Bioreactor. The radial flow bioreactor is designed to model the near well-bore environment and consists of four concentric polyvinyl chloride (PVC) pipe sections sealed with grooved top and bottom plates (Figure 1). The reactor is the same as was used in a previous study to detect biofilm growth in sand using the same NMR logging tool. ${ }^{21}$ In the current experiment, the height of the reactor was $50 \mathrm{~cm}$. The inner and outer pipes are solid while the two inner pipes are slotted to allow radial flow through the sand annulus between them. The inner and outer annuli are the influent and effluent reservoirs, respectively. The sand annulus measures $7.6 \mathrm{~cm}$ wide and was filled with $1 \mathrm{~mm}$ nominal quartz sand (2095 Granusil silica sand, Unimin Corp., Ottawa, MN). The liquid volume of the reactor is approximately $30 \mathrm{~L}$, including the sand pore volume and influent and effluent reservoirs.

Media and Injection Strategy. Two kinds of substrate media were used in this study, a bacterial growth medium (growth medium) and a calcite mineralization-promoting medium (calcium medium). Both were urea- and yeast extract-based ( $1 \mathrm{~g} / \mathrm{L}$ yeast extract (Arcos Organics, Gheel, Belgium), $20 \mathrm{~g} / \mathrm{L}$ urea, $1 \mathrm{~g} / \mathrm{L} \mathrm{NH}_{4} \mathrm{Cl}$, and $24 \mathrm{~g} / \mathrm{L} \mathrm{NaCl}$ ). The calcium medium contained an added $49 \mathrm{~g} / \mathrm{L} \mathrm{CaCl}_{2} \cdot 2 \mathrm{H}_{2} \mathrm{O}$. Commercial-grade chemicals were used for urea (Urea Fertilizer, Espoma, Millville, NJ), calcium chloride (various brands of commercial ice melt), and sodium chloride (Morton Table Salt, Chicago, IL). Media were mixed just prior to use in a nonsterile manner using tap water.

A pulsed-flow injection strategy promoted an even distribution of $\mathrm{CaCO}_{3}$ precipitation by balancing reaction and transport rates. ${ }^{31}$ Each $30 \mathrm{~L}$ pulse of substrate was pumped at a flow rate of $1 \mathrm{~L} / \mathrm{min}$, producing a pore velocity of approximately $0.4 \mathrm{~cm} / \mathrm{min}$ and ensuring that the fresh substrate would penetrate the full width of the sandpack. Calcium medium was injected four times per day during the biomineralization phase, Days 4-7. A $2 \mathrm{~h}$ batch reaction period (no flow) followed each injection of calcium medium. One pulse of growth medium was injected each evening to stimulate the bacteria for the following day's calcium medium injections. A $10 \mathrm{~L}$ brine rinse $(24 \mathrm{~g} / \mathrm{L} \mathrm{NaCl})$ was injected into the reactor first each morning to reduce mixing of the two substrate media in the influent reservoir and minimize clogging of the slotted pipe.

Bacterial Culture. The bacteria used in this experiment, Sporosarcina pasteurii (ATCC 11859), formerly known as Bacillus pasteurii, is widely used in laboratory experiments related to urea hydrolysis and biomineralization. ${ }^{8}$ S. pasteurii is a nonpathenogenic natural soil organism capable of producing relatively large amounts of the urease enzyme needed to catalyze urea hydrolysis. ${ }^{8}$ For the inoculum, $1 \mathrm{~mL}$ of frozen stock of $S$. pasteurii was cultured in $100 \mathrm{~mL}$ of growth medium on a shaker table at $150 \mathrm{rpm}$ for $24 \mathrm{~h}$. The $100 \mathrm{~mL}$ culture was then added to $10 \mathrm{~L}$ of fresh growth medium and mixed on a stir plate at $1150 \mathrm{rpm}$ for $24 \mathrm{~h}$. Finally, the $10 \mathrm{~L}$ culture was added to $20 \mathrm{~L}$ of fresh growth medium and mixed as before to produce a final inoculum volume of $30 \mathrm{~L}$. No attempt was made to maintain a monoculture in the inoculum or in the reactor.

The reactor was inoculated by first injecting $5 \mathrm{~L}$ of fresh growth medium to condition the reactor at a flow rate of $1 \mathrm{~L} /$ min, followed by the $30 \mathrm{~L}$ inoculum. An additional $5 \mathrm{~L}$ of fresh growth medium was injected last. Bacteria were allowed to attach to the sand for approximately $15 \mathrm{~h}$ with no flow before the first injection of calcium medium. There was no calcium present in the reactor during the 3 day control period or during inoculation. The initial period was used as the control. Note that previous experiments have shown no permeability reduction was achieved when urea and calcium containing solutions were injected into glass bead filled columns that were not inoculated with ureolytic microbes. ${ }^{32}$

NMR Measurements. Low-field NMR measurements typically consist of repeated scans which are stacked and averaged to reduce noise in the data. In this study, two 

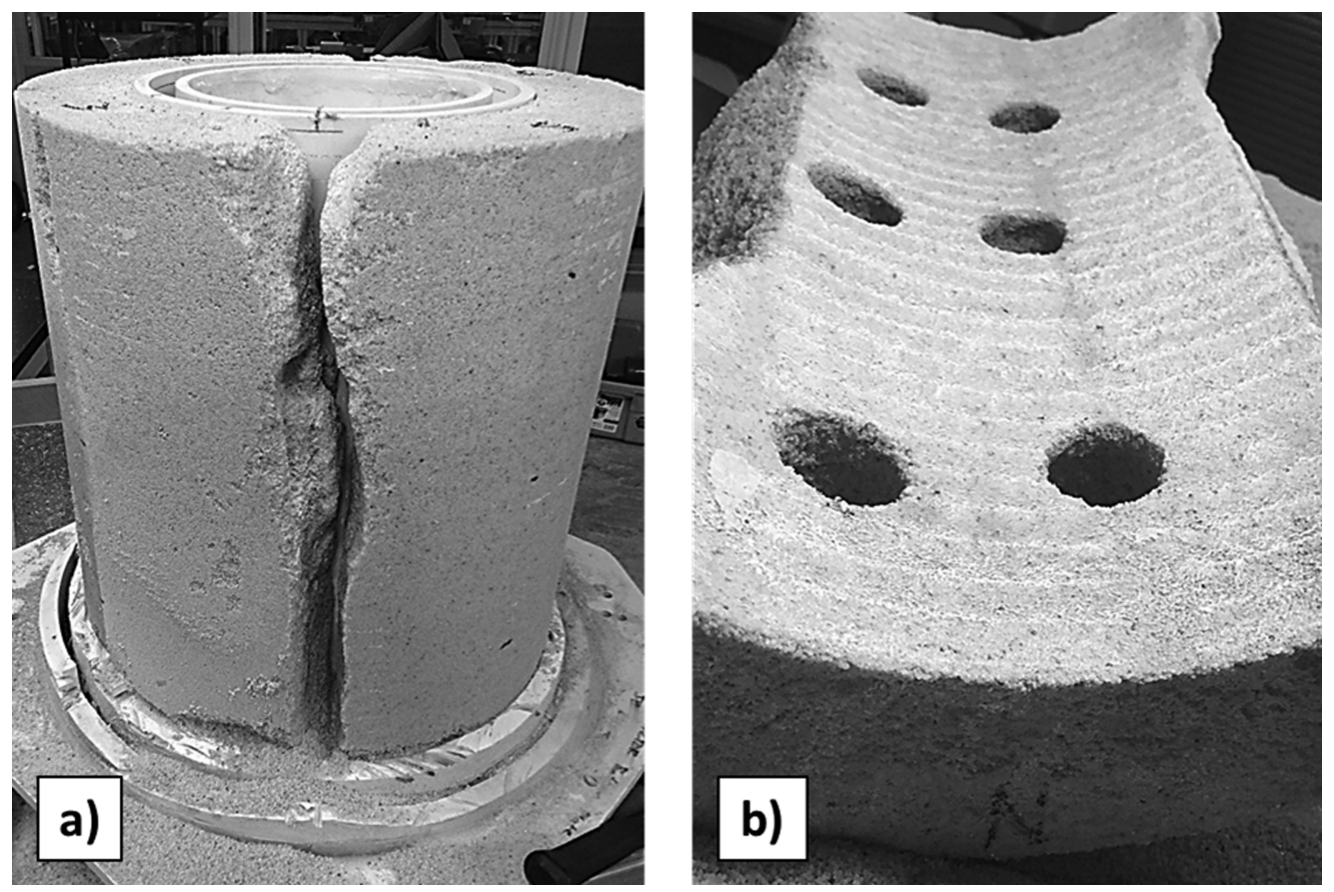

Figure 2. Biomineralized sand annulus was destructively sampled to quantify $\mathrm{CaCO}_{3}$ precipitation. (a) The outer pipes of the bioreactor were cut away to expose the biomineralized sand annulus. A saw was used to cut the annulus into quarters, producing the large crack shown here. (b) Six radial core samples were collected from each quarter.

experiments were conducted sequentially and together constitute one CPMG scan for measurement of $T_{2}$ relaxation. Experiment 1 collects $T_{1}$-weighted fast-decaying signal $\left(t_{\mathrm{E}}=1.3\right.$ $\mathrm{ms}, T_{\mathrm{r}}=800 \mathrm{~ms}, 54$ echoes, 360 averages). Experiment 2, on the other hand, collects the signal from spins with longer relaxation times $\left(t_{\mathrm{E}}=1.3 \mathrm{~ms}, T_{\mathrm{r}}=5000 \mathrm{~ms}, 334\right.$ echoes, 60 averages). All NMR measurements were collected under no flow conditions. Measurements during the control period, Days 1-3, consisted of 24 CPMG scans. Three (3) CPMG scans were stacked and averaged for each daily measurement during the biomineralization phase, Days 4-8, because of the timing of repeated substrate injections on a $2 \mathrm{~h}$ cycle. Data presented here was collected with a noise level of approximately $1.4 \%$.

As only one tool was available on loan for a limited period, it was not possible to run replicate experiments. However, previous work with this tool ${ }^{21,22}$ has allowed multiple experimental runs while monitoring biofouling both in a sand pack and in the subsurface. The tool's performance has been consistent and repeatable.

Sampling. Influent and effluent samples were collected for each injection of brine, calcium medium, and growth medium. The sample $\mathrm{pH}$ was measured shortly after collection; then, the sample was filtered $(0.2 \mu \mathrm{m}$ membrane, VWR International, Radnor, PA) and refrigerated for later analysis with the Jung Assay $^{33}$ to evaluate the sample urea concentration. After the final measurement on Day 8, the reactor was drained and destructively sampled. The outer pipe was cut away in sections, leaving the biomineralized sand annulus exposed for sampling (Figure 2). Twenty-four (24) cores were collected: 2 radial cores of approximately $1 \mathrm{in}$. diameter $(2.5 \mathrm{~cm})$ and $3 \mathrm{in}$. length $(7.5 \mathrm{~cm})$ at each of 3 depths were sampled in 4 orthogonal directions. Each core sample was divided into 3 subsamples which were then weighed and subjected to nitric acid digestion to remove the solid precipitates. The liquid was extracted for calcium content analysis by ICP-MS using an Agilent 7500ce
(Santa Clara, CA) with a collision cell (helium mode) and a certified environmental calibration standard from CPI International (product number 4400-12 1116NCO2). Additionally, micrograph images were acquired using a Zeiss Supra 55VP scanning electron microscope (Zeiss, USA). Biomineralized sand samples from the reactor and control sand samples were sputter coated with iridium, and high-resolution images were taken at $1.0 \mathrm{kV}$ at a working distance of 3-4 $\mathrm{mm}$.

\section{RESULTS AND DISCUSSION}

The influence of $\mathrm{CaCO}_{3}$ precipitation on the NMR signal response is reflected in the daily signal decay curves and resulting $T_{2}$ distributions where significant changes were observed over time. Representative data, collected on Days 2, 4,6 , and 8, are presented in Figure 3; the top panel shows fits to recorded signal decay curves, and the bottom panel presents the $T_{2}$ distributions for those decay curves. First, we will address the change in water content which corresponds to a drop in the porosity of the sandpack. Then, we will discuss the relaxation distributions, which give insight into changes in the relaxation mechanism.

Water Content and Porosity. Decreasing signal amplitude over time is an indication of $\mathrm{CaCO}_{3}$ precipitation, since $\mathrm{CaCO}_{3}$ will displace water in the pore volume. During the control period, the initial porosity indicated by the NMR-measured total water content was approximately $30 \%$ which is slightly less than the $35-39 \%$ expected from a sand pack with relatively uniform grains. The observation of entrained air leaving the system after the first flow pulse following inoculation, and the subsequent increase in the water content signal on Day 4, leads us to conclude that the sand pack was not fully saturated during the control period. This also explains why the measured water content value of $\sim 30 \%$ is less than the expected value of $35-$ $39 \%$. The NMR-measured total water content in the reactor decreased to approximately $76 \%$ of its original value between 

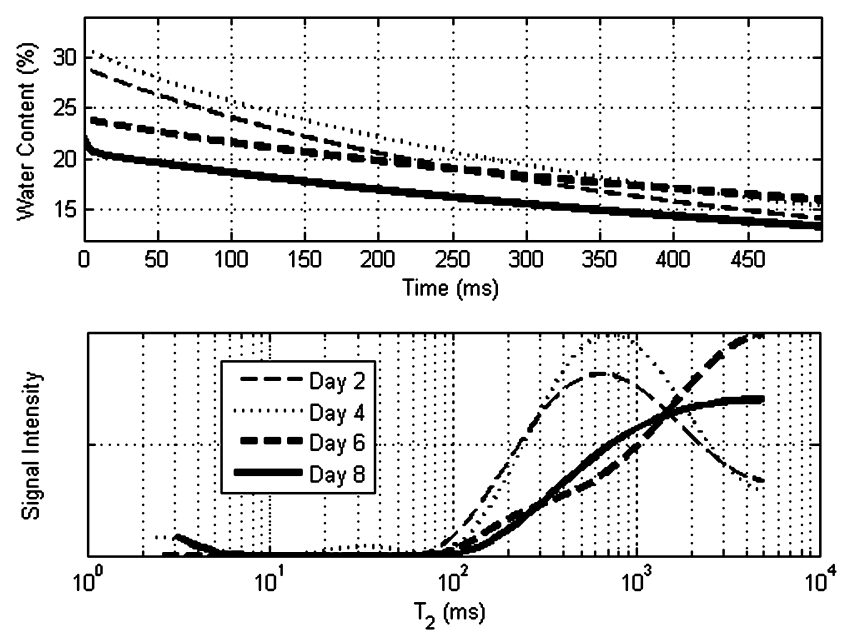

Figure 3. Signal decay curves (top) and the corresponding $T_{2}$ distributions (bottom) are shown with each curve representing a day. Day 2 occurred during the control period. Inoculation occurred on Day 3 (not shown). The calcium media injections occurred between Day 4 and 7. The Day 8 data was collected prior to flushing the reactor with brine and destructively sampling. Both graphs show fits to the raw data.

the control period (Day 2 data) and the end of the biomineralization phase on Day 8 (Figure 3, top panel, and Figure 4). This reduction in total water content indicates that

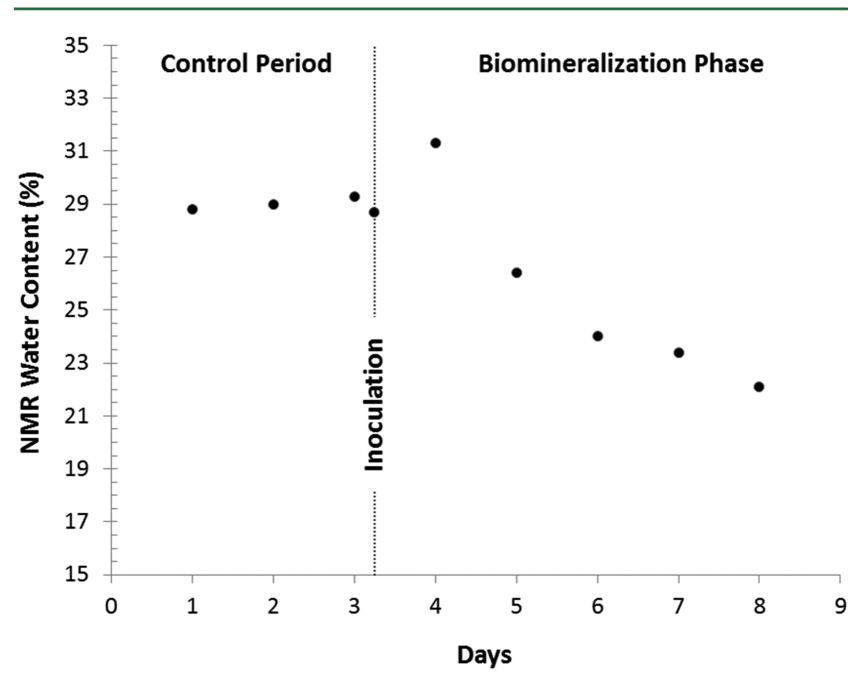

Figure 4. Measured total water content in the radial flow reactor decreased from approximately $29 \%$ during the control period Days 13 to approximately $22 \%$ by Day 8 . Note that the increase on Day 4 is real and well outside expected error bounds. The increase follows the observation of entrained air leaving the bioreactor, indicating the desired fully saturated state may not have been obtained until after the control period.

the pore volume within the reactor decreased significantly during the biomineralization phase. If we consider Day 4 to represent full saturation, then the NMR-estimated porosity reduction is $70 \%$ of the initial value, indicating the sensitivity of the NMR measurement to partial saturation.

$\mathrm{CaCO}_{3}$ formation was confirmed by scanning electron microscopy (SEM). There appeared to be a relatively uniform $\mathrm{CaCO}_{3}$ coating on the sand samples viewed with SEM. Figure 5 shows an SEM micrograph showing the crystals formed on a grain of sand from the reactor (a) and the surface of a control sand grain (b). The surface of the $\mathrm{CaCO}_{3}$-encrusted sand reveals microscale cavities and pores between crystals. No bacteria were visible in the sand samples viewed with SEM; it is most likely that the cells are entombed within the crystals.

Several methods were applied to estimate the volume of $\mathrm{CaCO}_{3}$ formed in the reactor in order to independently determine the reduction in pore volume achieved. These methods include a mass balance on urea, ICP-MS detection of $\mathrm{Ca}^{2+}$, and gravimetric methods. An initial porosity estimate of $37 \%$, typical for the sand in the reactor, was used in these calculations. Because the reactor was not fully saturated during the control period, the total porosity is greater than the NMR water content. The results of these three methods are in good agreement with each other and support the NMR data showing a significant pore volume reduction due to calcite precipitation.

Mass Balance on Urea. Influent and effluent samples of each pulse of media were analyzed using the Jung Assay ${ }^{33}$ to quantify the urea content. A mass balance on urea showed that approximately $4.2 \mathrm{~kg}$ of urea was consumed within the reactor, stoichiometrically producing approximately $6.9 \mathrm{~kg}$ of calcite. This mass of calcite would occupy at maximum approximately $15 \%$ of the pore space in the sand annulus. Since $\mathrm{CaCO}_{3}$ also formed in the tubing and on the reactor walls, we consider the urea mass balance method to provide an approximation of the upper bound of $\mathrm{CaCO}_{3}$ volume.

ICP-MS. ICP-MS was used to measure the concentration of $\mathrm{Ca}^{2+}$ in the acid extraction liquid from 24 samples of biomineralized sand from the reactor. A mean value of 9.36 $\mathrm{g} / \mathrm{L} \mathrm{Ca}^{2+}$ was obtained with a sample standard deviation of 1.89 $\mathrm{g} / \mathrm{L}$, which equates to an average total mass of $6.3 \mathrm{~kg} \mathrm{CaCO}$ within the sand. The ICP-MS data translates to an average pore volume reduction of approximately $12 \%( \pm 2.4 \%)$.

Gravimetric Method. The 24 sand samples were also weighed before and after the acid digestion removed the precipitate, resulting in an average mass of calcite of $63.6 \mathrm{mg}$ $\mathrm{CaCO}_{3} / \mathrm{g}$ sand with a sample standard deviation of $13 \mathrm{mg} / \mathrm{g}$. By this method, the average total mass within the sandpack was $5.5 \mathrm{~kg}$ of calcite. The gravimetric method indicates that approximately $11 \%( \pm 2.2 \%)$ of the pore space in the sand annulus was occupied by $\mathrm{CaCO}_{3}$ at the end of the experiment. Unlike the mass balance method, ICP-MS and gravimetry account only for $\mathrm{CaCO}_{3}$ attached to the sand. On the basis of these complementary and independent methods, we estimate that $\mathrm{CaCO}_{3}$ occupied approximately $11-12 \%$ of the pore space in the sandpack by Day 8 of the experiment.

This estimated porosity reduction is significantly higher than those previously reported in other NMR/MICP studies. ${ }^{10,20}$ The pulsed flow injection strategy used here promotes relatively uniform $\mathrm{CaCO}_{3}$ precipitation, as evidenced by the small standard deviation of the samples collected from the reactor. The uniform calcite precipitation implies spatially uniform porosity reduction. Consequently, only an insignificant reduction in permeability was observed in this study.

Compared to the methods described above which found final porosity to be approximately $88 \%$ of the original value, NMR measurements of water content overestimate the porosity reduction achieved. Final NMR water content was $76 \%$ of the initial value, or $70 \%$ of the Day 4 value. The overestimation can be attributed to carbon dioxide $\left(\mathrm{CO}_{2}\right)$ gas production inside the reactor. The excess $\mathrm{CO}_{2}$ produced by microbial oxidation of the yeast extract in the substrate can be trapped in the reactor pore spaces, displacing water and reducing signal amplitude 

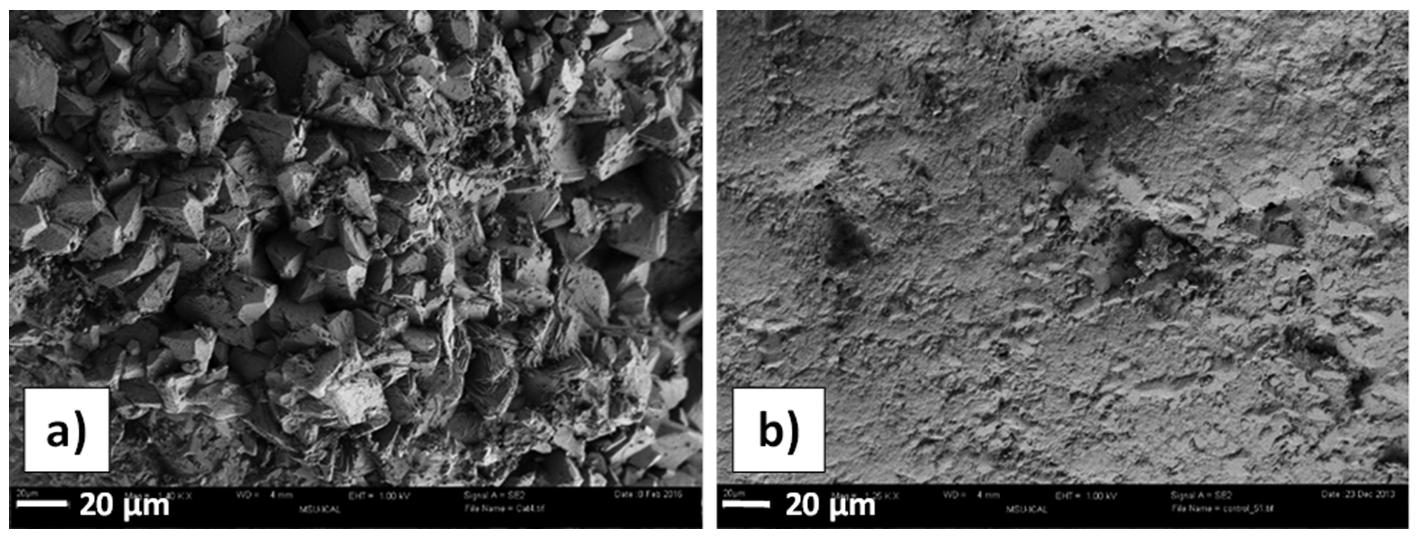

Figure 5. SEM image of (a) $\mathrm{CaCO}_{3}$ crystals attached to a grain of sand from the reactor following 4 days of MICP and (b) control sand without $\mathrm{CaCO}_{3}$. Scale bar is $20 \mu \mathrm{m}$. Note that the $\mathrm{CaCO}_{3}$ crystals completely cover the sand surface and the sand is not visible in (a), whereas in (b) the smooth sand surface is observed.

without changing the pore geometry. Gas formation was also observed in previous NMR studies of MICP. ${ }^{10,20}$ Furthermore, signal decaying in the interval before the first echo acquisition will underestimate the water content and may explain in part the NMR overestimation of porosity reduction.

Relaxation. The tall initial peak (Day 2 data) in the bottom panel of Figure 3, centered about approximately 600-700 ms and associated with water in large pores, first increases and then decreases in amplitude over time as the biomineralization phase proceeds. At the same time, there is an increase in both the occurrence of very fast $T_{2}$ relaxation times less than $10 \mathrm{~ms}$ and an increase in the proportion of spins experiencing very long relaxation times, greater than $1000 \mathrm{~ms}$. At the left-hand limit of the $T_{2}$ distribution (Figure 3, bottom), the NMR logging tool cannot capture NMR signal that decays faster than the measurement echo time $\left(t_{\mathrm{E}}=1.3 \mathrm{~ms}\right)$. We note that, since the time of this study, the echo time of the Javelin tool has been reduced to $0.7 \mathrm{~ms}$. At the right of the distribution, signals with $T_{2}$ between 1 and $5 \mathrm{~s}$ are not tightly resolved on the $T_{2}$-axis because the signal is sampled only to $500 \mathrm{~ms}$. However, the amplitude of these long signals is accurately measured (Figure 3 , top). By Day 8 of the experiment, the mean $\log T_{2}$ time of the distribution had increased to greater than $1000 \mathrm{~ms}$ from approximately $650 \mathrm{~ms}$ during the control period.

The data shows that $T_{2}$ relaxation in the macropores of the sandpack is more significantly affected by the reduction in $\rho$ than by the decrease in the macropore dimension. As seen in the SEM images (Figure 5a), the $\mathrm{CaCO}_{3}$ crystals are on the order of $10^{1} \mu \mathrm{m}$ thick. In a large pore on the order of $10^{2} \mu \mathrm{m}$ in diameter, there is a relatively minor change in pore dimension due to calcite precipitation. On the other hand, the relatively thin and uniform coating of $\mathrm{CaCO}_{3}$ crystals is sufficient to minimize molecular interactions between the pore fluid and paramagnetic species on the sand, making the surface much less likely to induce relaxation. The combination of a large change to $\rho$ and a small change to the pore size explains the lengthening of the overall mean $\log T_{2}$ relaxation time. At the same time, $\mathrm{CaCO}_{3}$ precipitation also creates micropores between and within the crystals. In these pores, the pore size effect dominates and $T_{2}$ relaxation occurs rapidly for the small population of spins within the crystals.

Previous NMR/MICP studies reporting the opposite ${ }^{20}$ or no relaxation effect ${ }^{10}$ are not at odds with this interpretation of the data. Both previous studies used smaller diameter $(\sim 100-250$ $\mu \mathrm{m}$ ) model porous media (borosilicate or polystyrene beads, respectively) with a low initial $\rho$ and small initial pore size, where we would expect more potential influence from a change in the pore geometry than from a reduction in $\rho$. We expect that, if $\mathrm{CaCO}_{3}$ precipitation had continued to progress in the current experimental system, the reduction in the macropore dimension would eventually become the dominate influence, driving relaxation times to decrease. Thus, the potential complexity of the relaxation response leaves open the possibility of different relaxation signatures in other porous materials where pore sizes or surface properties are more heterogeneous.

Our results show that changes in NMR signal response due to MICP include (1) a decrease in signal amplitude over time, indicating a reduction in porosity, and (2) a lengthening of the overall $T_{2}$ relaxation time in the quartz sand of the bioreactor. NMR measured water content in the reactor decreased to approximately $76 \%$ of the initial value, which corresponds well to the measured reduction in porosity to approximately $88 \%$ of the typical initial value. The extent of the decrease in porosity, and the corresponding minimal change in permeability, is related to the pulsed-flow injection strategy employed to achieve the MICP. $T_{2}$ relaxation distributions bifurcated from a single mode centered about approximately $650 \mathrm{~ms}$ during the control period into a very fast decaying population $\left(T_{2}\right.$ less than $10 \mathrm{~ms}$ ), associated with water in the porous $\mathrm{CaCO}_{3}$, and a larger population with relaxation times greater than $1000 \mathrm{~ms}$, corresponding to the bulk water in the large crystal-coated pores. Slower relaxation is caused by $\mathrm{CaCO}_{3}$ crystals on the mineral surface of the macropores shielding paramagnetic species from the pore fluid, reducing $\rho$ of the pore. In the $\mathrm{CaCO}_{3}$ micropores, the pore size effect dominated and enhanced relaxation. Future work will evaluate the NMR signal response to MICP in natural soils and porous rock where surface relaxivity and pore sizes are more heterogeneous. This study demonstrates that a NMR well-logging tool is sensitive to MICP and has potential as a sensor for biomineralization in field applications where optical or destructive monitoring methods are not possible. 


\section{ORCID}

Sarah L. Codd: 0000-0003-3859-8565

Notes

The authors declare no competing financial interest.

\section{ACKNOWLEDGMENTS}

This material is based upon work supported by the National Science Foundation Graduate Research Fellowship Program under Grant No. DGE-1049562 and by the Department of Energy under Grants No. DE-FE0024296 and DE-FG0211ER9002. Any opinions, findings, conclusions or recommendations expressed herein are those of the authors and do not necessarily reflect the views of the Department of Energy (DOE). The authors also thank Ana Paula Coelho, Drew Norton, Laura Dobeck, and Randy Hiebert at the Center for Biofilm Engineering.

\section{REFERENCES}

(1) Stoodley, P.; Sauer, K.; Davies, D. G.; Costerton, J. W. Biofilms as complex differentiated communities. Annu. Rev. Microbiol. 2002, 56, 187-209.

(2) Beech, I. B.; Sunner, J. Biocorrosion: towards understanding interactions between biofilms and metals. Curr. Opin. Biotechnol. 2004, 15 (3), 181-6.

(3) Costerton, J. W.; Stewart, P. S.; Greenberg, E. P. Bacterial biofilms: A common cause of persistent infections. Science 1999, 284 (5418), 1318-1322.

(4) de Kreuk, M.; Heijnen, J. J.; van Loosdrecht, M. C. M. Simultaneous COD, nitrogen, and phosphate removal by aerobic granular sludge. Biotechnol. Bioeng. 2005, 90 (6), 761-769.

(5) Cunningham, A. B.; Sharp, R. R.; Hiebert, R.; James, G. Subsurface biofilm barriers for the containment and remediation of contaminated groundwater. Biorem. J. 2003, 7 (3-4), 151-164.

(6) Decho, A. W. Overview of biopolymer-induced mineralization: What goes on in biofilms? Ecol. Eng. 2010, 36 (2), 137-144.

(7) Ferris, F. G.; Phoenix, V.; Fujita, Y.; Smith, R. W. Kinetics of calcite precipitation induced by ureolytic bacteria at 10 to 20 degrees $\mathrm{C}$ in artificial groundwater. Geochim. Cosmochim. Acta 2004, 68 (8), $1701-1710$.

(8) Phillips, A. J.; Gerlach, R.; Lauchnor, E.; Mitchell, A. C.; Cunningham, A. B.; Spangler, L. Engineered applications of ureolytic biomineralization: a review. Biofouling 2013, 29 (6), 715-733.

(9) Stocks-Fischer, S.; Galinat, J. K.; Bang, S. S. Microbiological precipitation of CaCO3. Soil Biol. Biochem. 1999, 31 (11), 1563-1571.

(10) Fridjonsson, E. O.; Seymour, J. D.; Schultz, L. N.; Gerlach, R.; Cunningham, A. B.; Codd, S. L. NMR measurement of hydrodynamic dispersion in porous media subject to biofilm mediated precipitation reactions. J. Contam. Hydrol. 2011, 120-121, 79-88.

(11) De Muynck, W.; De Belie, N.; Verstraete, W. Microbial carbonate precipitation in construction materials: A review. Ecol. Eng. 2010, 36 (2), 118-136.

(12) DeJong, J. T.; Mortensen, B. M.; Martinez, B. C.; Nelson, D. C. Bio-mediated soil improvement. Ecol. Eng. 2010, 36 (2), 197-210.

(13) van Paassen, L. A.; Ghose, R.; van der Linden, T. J. M.; van der Star, W. R. L.; van Loosdrecht, M. C. M. Quantifying Biomediated Ground Improvement by Ureolysis: Large-Scale Biogrout Experiment. J. Geotech. Geoenviron. Eng. 2010, 136 (12), 1721-1728.

(14) Mortensen, B. M.; Haber, M. J.; DeJong, J. T.; Caslake, L. F.; Nelson, D. C. Effects of environmental factors on microbial induced calcium carbonate precipitation. J. Appl. Microbiol. 2011, 111 (2), 338-349.

(15) Mitchell, A. C.; Dideriksen, K.; Spangler, L. H.; Cunningham, A. B.; Gerlach, R. Microbially Enhanced Carbon Capture and Storage by Mineral-Trapping and Solubility-Trapping. Environ. Sci. Technol. 2010, 44 (13), 5270-5276.

(16) Phillips, A. J.; Cunningham, A. B.; Gerlach, R.; Hiebert, R.; Hwang, C.; Lomans, B. P.; Westrich, J.; Mantilla, C.; Kirksey, J.;
Esposito, R.; Spangler, L. Fracture Sealing with Microbially-Induced Calcium Carbonate Precipitation: A Field Study. Environ. Sci. Technol. 2016, 50 (7), 4111-4117.

(17) Phillips, A. J.; Lauchnor, E.; Eldring, J.; Esposito, R.; Mitchell, A. C.; Gerlach, R.; Cunningham, A. B.; Spangler, L. H. Potential CO2 Leakage Reduction through Biofilm-Induced Calcium Carbonate Precipitation. Environ. Sci. Technol. 2013, 47 (1), 142-149.

(18) Behroozmand, A. A.; Keating, K.; Auken, E. A Review of the Principles and Applications of the NMR Technique for Near-Surface Characterization. Surveys in Geophysics 2015, 36 (1), 27-85.

(19) Walsh, D.; Turner, P.; Grunewald, E.; Zhang, H.; Butler, J. J.; Reboulet, E.; Knobbe, S.; Christy, T.; Lane, J. W.; Johnson, C. D.; Munday, T.; Fitzpatrick, A. A small-diameter NMR logging tool for groundwater investigations. Groundwater 2013, 51 (6), 914-926.

(20) Sham, E.; Mantle, M. D.; Mitchell, J.; Tobler, D. J.; Phoenix, V. R; Johns, M. L. Monitoring bacterially induced calcite precipitation in porous media using magnetic resonance imaging and flow measurements. J. Contam. Hydrol. 2013, 152, 35-43.

(21) Kirkland, C. M.; Hiebert, R.; Phillips, A.; Grunewald, E.; Walsh, D. O.; Seymour, J. D.; Codd, S. L. Biofilm Detection in a Model WellBore Environment Using Low-Field Magnetic Resonance. Groundwater Monitoring \& Remediation 2015, 35, 36-44.

(22) Kirkland, C. M.; Herrling, M. P.; Hiebert, R.; Bender, A. T.; Grunewald, E.; Walsh, D. O.; Codd, S. L. In Situ Detection of Subsurface Biofilm Using Low-Field NMR: A Field Study. Environ. Sci. Technol. 2015, 49 (18), 11045-11052.

(23) Grunewald, E.; Knight, R. A laboratory study of NMR relaxation times in unconsolidated heterogeneous sediments. Geophysics 2011, 76 (4), G73-G83.

(24) Kleinberg, R. L.; Horsfield, M. A. Transverse relaxation processes in porous sedimentary rock. J. Magn. Reson. 1990, 88 (1), 9-19.

(25) Kleinberg, R. L.; Kenyon, W. E.; Mitra, P. P. Mechanism of NMR relaxation of fluids in rock. J. Magn. Reson., Ser. A 1994, 108, 206-214.

(26) Bryar, T. R.; Daughney, C. J.; Knight, R. J. Paramagnetic effects of iron(III) species on nuclear magnetic relaxation of fluid protons in porous media. J. Magn. Reson. 2000, 142 (1), 74-85.

(27) Foley, I.; Farooqui, S. A.; Kleinberg, R. L. Effect of paramagnetic ions on NMR relaxation of fluids at solid surfaces. J. Magn. Reson., Ser. A 1996, 123 (1), 95-104.

(28) Kenyon, W. E.; Kolleeny, J. A. NMR surface relaxivity of calcite with adsorbed Mn2+. J. Colloid Interface Sci. 1995, 170 (2), 502-514.

(29) Coates, G. R.; Marschall, D.; Mardon, D.; Galford, J. A new characterization of bulk-volume irreducible using magnetic resonance. Log Analyst 1998, 39 (1), 51-63.

(30) Zhang, Q.; Lo, S. W.; Huang, C. C.; Hirasaki, G. J.; Kobayashi, R.; House, W. V. Some exceptions to default NMR rock and fluid properties. In Transactions of the SPWLA Annual Logging Symposium (Society of Professional Well Log Analysts), 1998; pp FF1-FF14.

(31) Ebigbo, A.; Phillips, A.; Gerlach, R.; Helmig, R.; Cunningham, A. B.; Class, H.; Spangler, L. H. Darcy-scale modeling of microbially induced carbonate mineral precipitation in sand columns. Water Resour. Res. 2012, 48, W07519.

(32) Wheeler, L. A. Establishment of ureolytic biofilms and their influence on the permeability of pulse-flow porous media column systems. Master's Thesis, Montana State University, Bozeman, MT, 2009.

(33) Jung, D.; Biggs, H.; Erikson, J.; Ledyard, P. U. New colorimetric reaction for endpoint, continuous-flow and kinetic measurement of urea. Clin. Chem. 1975, 21 (8), 1136-1140. 